\title{
DRY EYE IN PRESENT SCENARIO
}

\author{
Chandni Karole ${ }^{1}$, Vandana Telgote 2
}

${ }^{1}$ Assistant Professor, Department of Ophthalmology, Index Medical College Hospital and Research Centre, Indore. ${ }^{2}$ Associate Professor, Department of Ophthalmology, Index Medical College Hospital and Research Centre, Indore.

ABSTRACT

\section{PURPOSE}

To compare which test modality is better and less time consuming for diagnosis of dry eye.

\section{MATERIAL AND METHOD}

The present study was conducted on 100 subjects in Department of Ophthalmology, Index Medical College and Hospital, Indore (MP), from July 2015 to December 2015. After routine ophthalmological examination, Tear film Breakup Time (TBUT), Schirmer's Test, Fluorescein staining, Rose Bengal staining tests were performed on patients and results were analysed.

\section{OBSERVATIONS}

A total of 100 subjects participated in the study; 48 (48\%) were male and 52 (52\%) were female (0.9:1). The mean Ocular Surface Disease Index (OSDI)-37.12 \pm 19.05 (Range 4-75), mean TBUT 11.37 \pm 3.69 seconds (Range 4-18 seconds), and Schirmer's test scores were calculated to be mean $11.87 \pm 5.04$ (Range 27). The mean time taken to undertake the OSDI questionnaire was significantly lower than for the TBUT and Schirmer's test $(\mathrm{P}<0.05)$ and the mean time taken for the TBUT was significantly lower than for Schirmer's test $(\mathrm{P}<0.05)$.

\section{CONCLUSION}

Diagnosis of dry eye might be strengthened if the OSDI and TBUT are both performed; these together would be less time consuming than a Schirmer's test alone.

\section{KEYWORDS}

Dry Eye, Tear Film Breakup Time, Schirmer Test, Ocular Surface Disease Index.

HOW TO CITE THIS ARTICLE: Karole C, Telgote V. Dry eye in present scenario. J. Evolution Med. Dent. Sci. 2016;5(24):1273-1275, DOI: $10.14260 /$ jemds/2016/298

\section{INTRODUCTION}

Dry eye is a disorder of the tear film, which occurs due to tear deficiency or excessive tear evaporation. It causes damage to the interpalpebral ocular surface and is associated with a variety of symptoms reflecting ocular discomfort.

The precorneal tear film is an essential component of the ocular surface and can be subdivided into an anterior lipid layer, a middle aqueous layer and an innermost mucin layer. These layers are produced by the Meibomian glands, the lacrimal gland and goblet cells of the conjunctiva, respectively. The tear film lubricates the eye, maintains nutrition and oxygenation of ocular structures acts as a refractive component and helps remove debris from the ocular surface.

In terms of tear production, dry eye can be divided into tear deficient and evaporative types. Tear deficiency dry eye can further be subdivided into non-Sjogren's syndrome and Sjogren's syndrome, which is an autoimmune disease associated with lacrimal and salivary gland lymphocytic infiltration. Evaporative dry eye can be divided into Meibomian Gland Disease (MGD) and exposure-related dry eye.

Financial or Other, Competing Interest: None.

Submission 01-02-2016, Peer Review 06-03-2016,

Acceptance 11-03-2016, Published 22-03-2016.

Corresponding Author:

Dr. Chandni Karole,

363, At Post Khalwa,

Khalwa Taluk,

Khandwa Dist.-450117,

Madhya Pradesh.

E-mail: dr.chandni777@gmail.com

DOI: $10.14260 /$ jemds $/ 2016 / 298$
In yet another group of patients, mucin deficiency due to Stevens-Johnson syndrome or ocular cicatricial pemphigoid is the underlying mechanism of dry eye. Because of different age distributions of the population, definitions of dry eye and methodologies, different studies have reported various prevalence rates of dry eye disease ranging from 5.5\% to $37.7 \%$. It can affect any race and is more common in women.1-5

\section{MATERIAL AND METHOD}

The present study was conducted on 100 subjects in Department of Ophthalmology, Index Medical College and Hospital, Indore (MP) from July 2015 to December 2015. The patients were analysed in accordance with demographic profile, predisposing factor, questionnaire, Tear Film Breakup Time (TFBUT), Schirmer's test, fluorescein staining, Rose Bengal staining after routine ophthalmological examination.

\section{Ophthalmologic Examination and Measurements}

Subjects underwent a detailed ophthalmic examination including best-corrected visual acuity, intraocular pressure measurement with noncontact tonometer, anterior segment and fundus examination with a slit-lamp and biomicroscope.

Symptoms and signs do not always correlate well with each other in many patients, so Ohashi et al. suggested that a combination of

1. Dry eye symptoms,

2. Suggestive findings on Schirmer $(<5 \mathrm{~mm}$ wetting after 5 minutes) and TFBUT $<10 \mathrm{sec}$, 
3. Fluorescein and Rose Bengal staining $(>3+)$ would verify clinical dry eye.

To confirm a diagnosis of dry eye, certain tests are required in the clinical setting. Tear film stability can be assessed with the fluorescein tear film break-up time test (TBUT). This measures the interval in seconds between a complete blink and the first appearance of a dry spot or discontinuity in the precorneal film. Patients with TBUT less than 10 seconds are classified with clinical dry eye. If there is aqueous deficiency, the tear meniscus will appear to be thin, less than $1 \mathrm{~mm}$ in height. Another clinical method for assessing the severity of dry eye is ocular surface dye staining. Fluorescein and Rose Bengal stains can be used as diagnostic dyes. Fluorescein staining occurs when the epithelial barrier is disrupted and serves as a good test for evaluation of dry eye. Rose Bengal stains devitalized epithelial cells on the conjunctiva and serves a similar purpose. However, Rose Bengal causes transient irritation after instillation and may be less comfortable. Patients with dry eye syndrome can show signs of punctate epitheliopathy and even corneal abrasions.

Another important clinical test is the Schirmer test, which measures aqueous tear production. This test is easy to perform in clinical settings, but may be subject to errors. Strips of filter paper called Schirmer strips are placed on the lower lid inside the tarsal conjunctiva. The patient is allowed to blink normally and the tear strip is scored according to the degree it wets in 5 minutes. There are two ways to perform this test: (a) Without topical anaesthesia (Schirmer test I), which evaluates the ability of the ocular surface to respond to surface stimulation, and (b) Under topical anaesthesia (Schirmer test II) which evaluates basal tear secretion. Patients with tear soaking less than $10 \mathrm{~mm}$ are considered to have clinical dry eye and eyes with less than $5 \mathrm{~mm}$ wetting are diagnosed as severe dry eye.

\section{RESULTS}

A total of 100 subjects participated in the study; $48(48 \%)$ were male and 52 (52\%) were female (0.9:1).

\begin{tabular}{|c|c|c|c|c|c|}
\hline Age Groups & Females & $\mathbf{\%}$ & Males & $\mathbf{\%}$ & Total \\
\hline$<30$ & 12 & 23 & 20 & 42 & 32 \\
\hline $31-45$ & 14 & 27 & 9 & 19 & 23 \\
\hline $46-60$ & 21 & 40 & 11 & 23 & 32 \\
\hline $61-75$ & 5 & 10 & 8 & 17 & 13 \\
\hline Total & $\mathbf{5 2}$ & $\mathbf{1 0 0}$ & $\mathbf{4 8}$ & $\mathbf{1 0 0}$ & $\mathbf{1 0 0}$ \\
\hline \multicolumn{7}{|c|}{ Age and Sex Incidence } \\
\hline
\end{tabular}

The mean Ocular Surface Disease Index (OSDI)$37.12 \pm 19.05$ (Range 4-75), mean TBUT $11.37 \pm 3.69$ seconds (Range 4-18 seconds) and Schirmer's test scores were calculated to be mean 11.87 \pm 5.04 (Range 27).

\begin{tabular}{|c|c|c|c|}
\hline Gender & Mean & Std. Deviation & Range \\
\hline Female & 11.71 & 4.579 & 17 \\
\hline Male & 12.04 & 5.539 & 27 \\
\hline Total & $\mathbf{1 1 . 8 7}$ & $\mathbf{5 . 0 4 4}$ & $\mathbf{2 7}$ \\
\hline \multicolumn{4}{|c|}{ Average Value of Schirmer Test Sex Wise } \\
\hline
\end{tabular}

According to the TBUT results, $42 \%$ of the subjects were diagnosed as having dry eye, while when the cut-off OSDI score was accepted as $\geq 35,35 \%$ of the subjects were diagnosed as having dry eye. The mean time taken for performing each test was as follows: OSDI, 74.11 \pm 28.61 seconds (Range 15-138 seconds); TBUT, $215.14 \pm 65.27$ seconds (Range 120-480 seconds); Schirmer's test, $339.39 \pm 72.29$ seconds (Range 120 480 seconds). The mean time taken to undertake the OSDI questionnaire was significantly lower than for the TBUT and Schirmer's test $(\mathrm{P}<0.05)$ and the mean time taken for the TBUT was significantly lower than for Schirmer's test $(\mathrm{P}<0.05)$.

\section{DISCUSSION AND CONCLUSION}

DES is a common public health problem encountered by ophthalmologists; however, diagnosis is not straightforward. The symptoms and signs do not correlate well. Ocular symptoms may not occur despite reduction of tear production. Nichols et al. reported that there was a poor relationship between the symptoms and results of diagnostic tests in patients with DES. ${ }^{6}$ A subject may have no symptoms besides eye tiredness, even though the result of a Schirmer's test is below $5 \mathrm{~mm}$ or even zero.

In this study, we found that prevalence of dry eye was $13.8 \%$ and women were more likely to have dry eye than males (1:0.9) and also at early age of 45 yrs. than male (70 yr.). ${ }^{7,8}$ wherever $18 \%$ were positively stained with fluorescein and $8 \%$ with rose Bengal stain.

This study, we performed three tests for confirmation of the diagnosis. The OSDI results were well correlated with those of the TBUT; $35 \%$ of the subjects were diagnosed as having DES according to the results of the OSDI and $42 \%$ of the subjects according to results of the TBUT. The mean time required to undertake OSDI test was $78.15 \pm 26.61$ seconds and $218.22 \pm 71.37$ seconds for the TBUT. The mean time spent in taking both tests was significantly lower than that required for Schirmer's test. Thus, we consider that diagnosis might be strengthened if the OSDI and TBUT are both performed; these together would be less time consuming than a Schirmer's test alone.

Schirmer's test alone does not seem to be a good test for diagnosis of dry eye disease because reflex epiphora might result in the misdiagnosis of dry-eye patients as being normal. Singh Bhinder and Singh Bhinder reported that Schirmer's test results changed according to reflex epiphora, therefore there was no correlation with symptoms in DES. ${ }^{9}$ Reflex epiphora that developed during the Schirmer's test may be the underlying cause of the absence of correlation between the OSDI and Schirmer's test scores in our study.

\section{REFERENCES}

1. Moss SE, Klein R, Klein BE. Prevalence of and risk factors for dry eye syndrome. Arch Ophthalmol 2000;118(9):1264-8.

2. McCarty CA, Bansal AK, Livingston PM, et al. The epidemiol ogy of dry eye in Melbourne, Australia. Ophthalmology 1998;105(6):1114-9.

3. Schein OD, Munoz B, Tielsch JM, et al. Prevalence of dry eye among the elderly. Am J Ophthalmol 1997;124(6):723-8.

4. Schaumberg DA, Sullivan DA, Buring JE, et al. Prevalence of dry eye syndrome among US women. Am J Ophthalmol 2003;136(2):318-26.

5. Gayton JL. Aetiology, prevalence, and treatment of dry eye disease. Clin Ophthalmol 2009;3:405-12. doi: 10.2147/OPTH.S5555. 
6. Nichols KK, Nichols JJ, Mitchell GL. The lack of association between signs and symptoms in patients with dry eye disease. Cornea 2004;23(8):762-770.

7. The definition and classification of dry eye disease: report of the definition and classification subcommittee of the international dry eye workshop. Ocul Surf 2007;5(2):7592.
8. Smith JA. The epidemiology of dry eye disease: report of the epidemiology subcommittee of the international dry eye workshop. Ocul Surf 2007;5(2):93-107.

9. Singh Bhinder G, Singh Bhinder H. Reflex epiphora in patients with dry eye symptoms: role of variable time schirmer-1 test. Eur J Ophthalmol 2005;15(4):429-433. 